\title{
The Design and Realization of Users one-stop service platform Based on 3G Network
}

\author{
Li Zhuyan ${ }^{1, a}$, Zhou Guixian ${ }^{2, b}$ * \\ ${ }^{1}$ Information Institute GuiZhou College of Financial and Economic, Guiyang 550004, China \\ ${ }^{2}$ Information Institute GuiZhou College of Financial and Economic, Guiyang 550004, China \\ a2225837531@qq.com, bhouasp1978@126.com
}

Keywords: one-stop service platform; $3 \mathrm{G}$ network

Abstract. designed and implemented a set of typical education cloud platform system, the platform adopts the double virtual network architecture, the network is divided into two layers: the underlying real device based on virtual support platform, to build the business layer.

\section{Introduction}

With the continuous development of wireless communication technology and the growing popularity of $3 \mathrm{G}$ networks, the number of projects applying wireless video transmission to video surveillance is increasing rapidly, developing especially fast in recent years. The area that combines mobile communication networks and embedded intelligent processing technology will become the development mainstream in the future progress trend of wireless video surveillance.

Modern video monitoring technology is a set computer technology! Network technology and multimedia technology in the integration of integrated technology, its intuitive, convenient, information is rich in content and features, get the favour of people more and more in recent years, are widely used in industrial production, transportation, electricity, intelligent office buildings and other places, has gradually become an important technology of modern management and testing.

At present, the emerging of network video monitoring system based on embedded technology compared with traditional monitoring system has small volume, low cost, high stability, good real-time, etc; Based on domestic commercial operation has $3 \mathrm{~g}$ wireless video monitoring system has powerful wireless network transmission function, can be overcome by geographical location. Wiring cost and remote monitoring and other problems, application and development prospect is good. Therefore, research and develop a embedded network video monitoring system based on $3 \mathrm{~g}$ standard has important application significance[1].

"Seeanywhere" 3G network technology, ADSL network technology, H.264 video compression techniques for system integration, to provide customers with one-stop service platform, users can through the PC, the web, $3 \mathrm{~g}$ mobile phone a variety of clients to realize single sign-on, multipoint monitoring. Platform the H.264 compression technology to achieve small streaming media transmission, adaptive network design, can penetrate various firewalls and routers, but also solve the dynamic IP access problems caused by the ADSL network, etc. Perfect alarm function, can email, FTP, SMS, MMS, video telephone alarm. Operation module design, including user management, statistical analysis, prepaid phone billing function, income statistics, user behavior analysis.

\section{THE KEY TECHNOLOGY RESEARCH OF WIRELESS VIDEO SURVEILLANCE}

1)H. 264 compression technology and embedded applications

H.264 is a new generation of video compression standard, is expected in the next few years will become the industry's preferred video standard. This standard has been successfully applied to electronic products such as mobile phones and digital video players. For video surveillance industry, h. 264 can reduce the storage cost, improve the overall efficiency, so as to further expand the application potential of video monitoring[2]. 
H. 264 (sometimes referred to as the mpeg-4 part10 / AVC) is a license to use open standards, to support the most efficient video compression technology on the market today. In the case of not affect image quality, and the M - JPEG compared to the traditional standard of mpeg-4 part2, h. 264 encoder can make digital video file size reduced by more than $80 \%$ and $50 \%$ respectively. Being able to provide such strong compression capacity, h. 264 become extremely suitable for the compression of video monitoring application standards.

At the same level of the image quality, a 115 second video streaming bit rate under different video standards. In the absence of motion compensation, h. 264 encoder efficiency of at least three times higher than the mpeg- 4 encoder, 6 times higher than the M - JPEG encoder.

2) Database technology

In today's market, commercial database called the warlords regime. Since the $1970 \mathrm{~s}$, the relational model is raised, due to its outstanding advantages, quickly adopted by commercial database system. According to statistics, since $70 \mathrm{~s}$ of the development of new DBMS system, nearly ninety percent is a relational data model, which emerged many excellent commercial relational database management system. Small Foxpro database system, for example, the ACCESS and PARADOX and so on, large DB2 database system, INGRES, ORACLE, INFORMIX, SYBASE, SQL SERVER, etc. In the $80 \mathrm{~s}$ and $90 \mathrm{~s}$ is the age of RDBMS product development and competition. Various products has experienced from concentration to distribution, from stand-alone environment to the network environment, support from information management to the online transaction processing (OLTP), to the development of the on-line analytical processing (OLAP); Support for the relational model also gradually improve; To enhance the function of the system[3].

With not only the development of software technology, database technology has not only mature, database has been in the telecommunications, finance and other areas to get a number of applications.

\section{THE DESIGN AND REALIZATION OF THE PLATFORM}

According to the China telecom Shanghai research institute of the actual test results show that the domestic widely used mpeg-4 coding technique under the bandwidth of 3 megabits per second is short of standard definition of image quality, and h. 264 coding technique can provide under $2 \mathrm{~m}$ bandwidth requirements of image effect. Thus operators hope to introduce more advanced h. 264 coding technology, under the limited bandwidth resources to improve the quality of the image.

According to the application requirements, we can make full use of various methods, h. 264, the advantages of high compression ratio. At present, in order not to exceed the application rules of storage limit, people usually choose to limit frame rate or lower resolution, but it will have a negative impact to video images, fuzzy images or details is not clear. If used in such applications support h. 264 compression standard video monitoring equipment, you can get a higher frame rate and image resolution and many other advantages, which can output higher quality of image.

Overall structure of the system is shown in Figure 1. The system consists of three components: video server, transmission network and surveillance client[4]. Audio and video signal captured by microphones and cameras of the monitoring scene, as the input of the video server, is packaged into a compressed encoded audio and video streaming, and then sent to the public Internet by the $3 \mathrm{G}$ mobile communication module via the $3 \mathrm{G}$ network. The client receives the audio and video streaming from the public Internet and sends related command and control information over the network to the video server. If we combine the system with a storage server and a streaming media distribution server, a large-scale video surveillance system is then obtained. This article focuses on design and implementation of the transmission part of the system. 


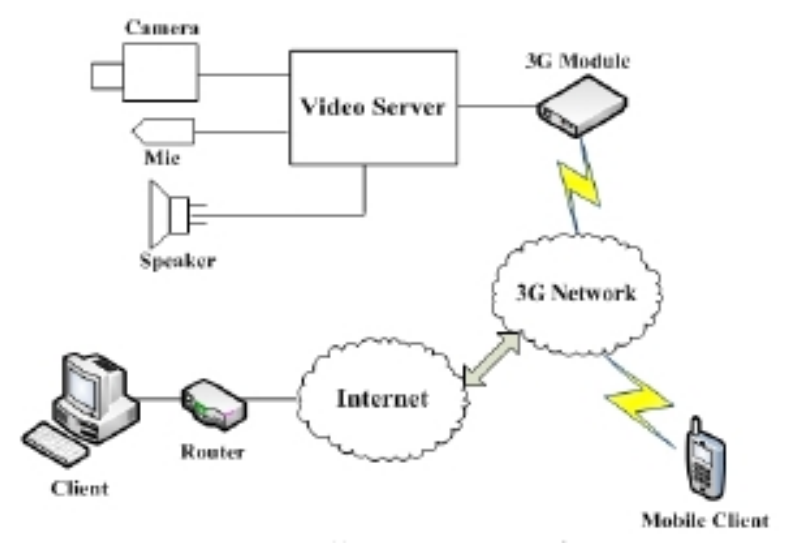

Figure 1. Overall structure of the system

1) Software design of the system transmission

The software of system transmission part requires the following functions: As a DTE, the video server uses the AT command to dial the SIM5218 after it is connected the SIM5218 through USB interface; And then the transmitting programs both running on the video server and the client are called to transmit the media streams and the control information between the server and the client. Therefore, the programs' development includes modification of the SIM5218's USB driver, both implementation of the video server dialing and the transmitting programs.

2) Monitoring the front-end design

I video compression format options "to choose the appropriate compression formats, large original digital video data compression to suitable for wireless network transmission of compressed video data;

I choose the right means of wireless Internet access;

I monitor the front-end hardware architecture, the choice of different architecture affect the performance of the system, volume, power consumption and cost, technical indicators;

I monitoring the front-end software platform of choice "is a necessary part of the system software, select the right software platform, including the operating system. Compile environment! Application! The underlying drivers, etc., for the smooth realization of the system play an important role.

3)Wireless transmission network design

We can use the existing IP network of wireless communication and have been commercially available: one for the cellular mobile communication network, and another for the satellite communication network, the IPSTAR transmission rate is high, but the cost is high, and is unfavorable to carry, therefore does not like the design to the requirement of the portable wireless video monitoring system, will be the focus of the wireless network design on the $3 \mathrm{~g}$ communication network in the field of cellular because wireless network transmission rate is low, instability and so on characteristics, therefore, in the network transmission through reasonable design of system software are needed to make up for the defect of wireless channels on the physical properties, to ensure that the video transmission to the requirement of real-time and accuracy.

4) implementation of the transmitting programs

At present, domestic operators of the $3 \mathrm{G}$ networks are China Unicom, China Mobile and ChinaTelecom. If the users don't submit a special application, the IP address they get is a private address by default when they use the access services of China Mobile and China Unicom's mobile network. If users use the IP address as a server's address, they cannot access the server from externalnet works. The system uses the $3 \mathrm{G}$ network services provided by the Unicom. To solve the problem that the client cannot access the video server's IP address, we redirect the connection. That is after two transmitting programs are implanted in the original server and the client respectively, the one running on the server first Initiate a connection. Coordination of the two transmitting programs isolates the original connection between the client and the server. At this point, the problem that server IP cannot be accessed from the external network has been solved. 
During the actual design of the transmitting programs, we need to obtain all the port numbers of the link between the client and the server. By analyzing the packages of the link we've intercepted, we find that two TCP ports are involved in the link. They are 80 and 22616. Because the realization of the transmitting programs running on the server is similar to the client's, only the flow charts of the server's transmitting program are illustrated.

\section{SUMMARY}

In this paper, The database in the large amount of data, high reliability, on the Internet technology and database technology, the combination of the development of a set of binding terminal 1 million, 500000 registered users the ability to operating video monitoring platform. This project needs to be Windows PC platforms, WINCE platform and Symbian platform of video decoding technology, video display technology, network transmission technology, the development of a set of multifunctional monitoring client.

\section{Acknowledgments}

This paper is supported by the Outstanding talents of science and technology education in guizhou provincial capital projects under Grant No.[2013]197

\section{References}

[1] Targets Detection and Analysis in Coastal Area Using Polarimetric SAR Images[A]. Proceedings of 2007 Asian and Pacific Conference on Synthetic Aperture Radar(APSAR 2007)[C]. 2007

[2] Ahmed Zainaldin,Ioannis Lambadaris,Biswajit Nandy. Adaptive Rate Control Low bit-rate Video Transmission over Wireless Zigbee Networks[A]. Proceedings of the Symposium on Communication Quality, Reliability, and Performance Modeling of ICC 2008[C]. 2008

[3] On Directional K-Coverage Analysis of Randomly Deployed Camera Sensor Networks[A]. Proceedings of the Symposium on Wireless Networking of ICC 2008[C]. 2008

[4] Li Nan,Wang Jing,Fang Haoshuai. Mobile Video Surveillance System Based on 3G and Hi3511 Chip[A]. Proceedings of 2012 2nd International Conference on Advanced Materials and Information Technology Processing(AMITP 2012) Volume 34[C]. 2012 